NBER WORKING PAPER SERIES

SALIENCE IN EXPERIMENTAL TESTS OF THE ENDOWMENT EFFECT

Pedro Bordalo

Nicola Gennaioli

Andrei Shleifer

Working Paper 17761

http://www.nber.org/papers/w17761

\author{
NATIONAL BUREAU OF ECONOMIC RESEARCH \\ 1050 Massachusetts Avenue \\ Cambridge, MA 02138 \\ January 2012
}

We are grateful to Dmitry Taubinsky for extremely helpful comments. Gennaioli thanks the Spanish Ministerio de Ciencia y Tecnologia (ECO 2008-01666 and Ramon y Ca jal grants), the Barcelona GSE Research Network and the Generalitat de Catalunya for financial support. Shleifer thanks the Kauffman Foundation for research support. The views expressed herein are those of the authors and do not necessarily reflect the views of the National Bureau of Economic Research.

NBER working papers are circulated for discussion and comment purposes. They have not been peerreviewed or been subject to the review by the NBER Board of Directors that accompanies official NBER publications.

(C) 2012 by Pedro Bordalo, Nicola Gennaioli, and Andrei Shleifer. All rights reserved. Short sections of text, not to exceed two paragraphs, may be quoted without explicit permission provided that full credit, including $\odot$ notice, is given to the source. 
Salience in Experimental Tests of the Endowment Effect

Pedro Bordalo, Nicola Gennaioli, and Andrei Shleifer

NBER Working Paper No. 17761

January 2012

JEL No. D03,D81

\section{ABSTRACT}

We provide a novel account of experimental evidence for the endowment effect using the salience mechanism (Bordalo, Gennaioli, and Shleifer, 2011). The two-stage procedure implemented in experiments implies that the endowed good and other goods are evaluated in different contexts. We describe conditions under which the standard effect occurs, but also account for recent evidence such as a reverse endowment effect for bads and a role for reference prices in modulating the WTA-WTP gap.

Pedro Bordalo

Department of Economics

Royal Holloway

University of London

Egham Hill, Egham, TW20 0EX

United Kingdom

pedro.bordalo@rhul.ac.uk

Nicola Gennaioli

CREI

Universitat Pompeu Fabra

Ramon Trias Fargas 25-27

08005 Barcelona (Spain)

ngennaioli@crei.cat
Andrei Shleifer

Department of Economics

Harvard University

Littauer Center M-9

Cambridge, MA 02138

and NBER

ashleifer@harvard.edu 


\title{
Salience in Experimental Tests of the Endowment Effect
}

\author{
Pedro Bordalo, Nicola Gennaioli, and Andrei Shleifer ${ }^{1}$
}

January 2012

\begin{abstract}
We provide a novel account of experimental evidence for the endowment effect using the salience mechanism (Bordalo, Gennaioli, and Shleifer, 2011). The two-stage procedure implemented in experiments implies that the endowed good and other goods are evaluated in different contexts. We describe conditions under which this leads to the standard effect, but also account for recent evidence such as a reverse endowment effect for bads and a role for reference prices in modulating the WTA-WTP gap.
\end{abstract}

\section{Introduction}

Starting with Jack Knetsch (1989), experiments on the "endowment effect" (Richard Thaler 1980) typically rely on a two-stage procedure. In the first stage, subjects are endowed with a good, such as a mug. In the second stage, the same subjects are given the opportunity to trade this good for another good of similar value, such as a pen. The endowment effect holds that very few subjects chose to trade, sometimes as few as $10 \%$. In related experiments, subjects state selling prices for their endowment, which are much higher than their buying prices for the same good. These patterns are hard to reconcile with standard choice theory, which predicts that about half the subjects would trade and that selling prices and buying prices are similar.

\footnotetext{
${ }^{1}$ Royal Holloway, University of London. CREI, Universitat Pompeu Fabra. Harvard University. We are grateful to Dmitry Taubinsky for extremely helpful comments. Gennaioli thanks the Spanish Ministerio de Ciencia y Tecnologia (ECO 2008-01666 and Ramon y Ca jal grants), the Barcelona GSE Research Network and the Generalitat de Catalunya for financial support. Shleifer thanks the Kauffman Foundation for research support.
} 
The common explanation of this evidence relies on Prospect Theory's loss aversion (Daniel Kahneman and Amos Tversky 1979). Because the pain of parting with the endowment looms larger in the decision maker's mind than the pleasure of acquiring a good of similar value (Kahneman, Knetsch, and Thaler 1991), a decision maker endowed with a mug is unwilling to trade it for a pen (or states a high selling price). ${ }^{2}$

Recent experimental evidence, however, suggests that loss aversion relative to expectations may not be the whole story. Perhaps the most revealing fact is that the endowment effect is sensitive to the type of goods involved and to the information available about them. Nathan Novemsky and Kahneman (2005) argue that the endowment effect should not arise in exchanges of identical goods. Lyle Brenner, Yuval Rottenstreich, Sanjay Sood and Baler Bilgin (2007) show that the pattern reverses in experiments concerning bads rather than goods, as decision makers become systematically eager to trade away their bad endowment. In experiments investigating the gap between selling and buying prices, the gap is sensitive to information about the market price of the endowment (Raymond Weaver and Shane Frederick 2011).

A common thread of these works is that contextual factors such as the nature of the goods involved or the information provided about market prices systematically affect the manifestation of the endowment effect in ways difficult to reconcile with standard accounts based on reference points and loss aversion. In this paper, we try to account for these findings by modeling the endowment effect as a form of context dependence, arising through the salience mechanism described in Bordalo, Gennaioli, and Shleifer (BGS 2011 a,b). As reviewed next, when a decision maker contemplates the options available to him, he focuses on - and gives disproportionate weight to - those features along which each option "stands out", or is salient, relative to the other options. In this way, a good's salient features, and thus its evaluation, depend on what it is compared to. The gist of our salience-based explanation of the endowment effect is that the two-stage procedure implemented in experiments (but perhaps also the experience of ownership in the real world) implies that the endowed good and the other goods are evaluated in different contexts.

\footnotetext{
${ }^{2}$ By modeling the reference point as expectations, Botond Koszegi and Matthew Rabin (2006) reconciled the endowment effect with the fact that people trade in large amounts if they expect to do so. See also John List (2003), Keith Ericson and Andreas Fuster (2011) and Ori Heffetz and List (2011).
} 
Specifically, after the decision maker is given the endowment good $e$, he values it in comparison with his status-quo of having nothing. In this context, what stands out are good $e$ 's best attributes. The decision maker overweights these attributes, which leads to an overvaluation of good $e$. This captures a perceptual "warm glow" induced by receiving a gift or getting ownership of an object (Tversky and Dale Griffin, 1991), driven by the decision maker's focus on that object's upside. In the second (trade) stage, the decision maker is given the option to trade the endowment $e$ for a new good $n$. Now the evaluation of $n$ is shaped not only by the backdrop of having nothing but also by the contrast between $n$ and $e$. When this contrast makes salient the new good's disadvantage relative to the endowment, the decision maker undervalues the new good and displays the endowment effect.

The critical asymmetry between the endowment and the new good comes from the warm glow of ownership: when the decision maker receives a mug, he focuses on its most valuable uses. These uses are still present in his mind when he considers exchanging the mug for a pen, so that the mug's valuation persists in the second stage. This logic yields the new predictions that the endowment effect should be reversed in the case of bads, should not arise when identical goods are exchanged, and that its manifestation should be sensitive to information about market prices.

\section{Salience}

Following BGS (2011b), we consider the simplest case of two-attribute goods, where a generic good $k$ is a two dimensional vector of qualities $\left(q_{1 k}, q_{2 k}\right) \in R^{2}$, and the decision maker's intrinsic utility is linear in the attributes, $v\left(q_{1 k}, q_{2 k}\right)=w_{1} q_{1 k}+w_{2} q_{2 k}$, where the weights $w_{i}$ sum up to 1 . The perceived value of the good, however, differs from its intrinsic value because the decision maker overweights the good's salient attribute at the expense of its non-salient attribute: if attribute $i$ is salient and attribute $j$ is not, then the decision maker evaluates good $k$ with weights given by

$$
\frac{w_{i}^{L T}}{w_{j}^{L T}}=\frac{1}{\delta} \cdot \frac{w_{i}}{w_{j}}, \quad w_{i}^{L T}+w_{j}^{L T}=1
$$


where $\delta \in(0,1]$ captures the degree to which the decision maker neglects non salient features. Referring to such neglect, we call our decision maker a "local thinker" (when $\delta=1$ the local thinker is a standard rational decision maker).

Which attribute is salient for good $k$ depends on two factors: the decision maker's consideration set $C$ and a salience function $\sigma: R^{2} \rightarrow R_{+}$. The set $C$ includes the goods considered by the decision maker when evaluating good $k$, and provides our measure of context. The salience of attribute $i=1,2$ for good $k$ is a function $\sigma\left(q_{i, k}, \bar{q}_{i}\right)$ that measures the extent to which the good's attribute $i$ "stands out" relative to its average value $\bar{q}_{i}$ in $C$. This intuition is in line with well established features of human perception: our visual apparatus automatically allocates scarce attentional resources to aspects of the environment that are most surprising or different from what is expected. To capture these features of perception, we assume that the salience function satisfies three properties: i) ordering: whenever an interval $[x, y]$ is contained in a larger interval $\left[x^{\prime}, y^{\prime}\right]$, we have $\sigma(x, y)<\sigma\left(x^{\prime}, y^{\prime}\right)$; ii) diminishing sensitivity (Weber's law): for all $x, y>0$ and any $\epsilon>0$, we have $\sigma(x+\epsilon, y+\epsilon)<\sigma(x, y)$; and iii) reflection: if $x, y, x^{\prime}, y^{\prime}>0$ then $\sigma(x, y)<\sigma\left(x^{\prime}, y^{\prime}\right)$ if and only if $\sigma(-x,-y)<\sigma\left(-x^{\prime},-y^{\prime}\right)$.

Following BGS (2011 b), we use a salience function symmetric and homogenous of degree zero $(\sigma(\alpha x, \alpha y)=\sigma(x, y)=\sigma(y, x)$ for all $\alpha>0$, with $\sigma(0,0)=0)$, which is sufficient to ensure diminishing sensitivity. A typical example is $\sigma(x, y)=|x-y| /(|x|+|y|)$. Due to ordering, salience $\sigma\left(q_{i k}, \bar{q}_{i}\right)$ increases with the distance $\left|q_{i k}-\bar{q}_{i}\right|$. Due to diminishing sensitivity and reflection $\sigma\left(q_{i k}, \bar{q}_{i}\right)$ decreases as $q_{i k}$ and $\bar{q}_{i}$ rise in absolute value.

\section{Of Mugs and Pens}

To formalize trade of mugs for pens, suppose that $q_{1}$ captures a good's "quality for drinking" while $q_{2}$ is its "quality for writing" (both measured in utils), and that the decision maker puts equal weight on both attributes, $w_{1}=w_{2}=1 / 2$. A $\operatorname{mug} M$ is a $\operatorname{good}\left(q_{M}, 0\right)$, a pen $P$ is a good $\left(0, q_{P}\right)$, where the zeroes capture the fact that experiments involve no writing mugs or drinking pens. ${ }^{3}$ Suppose further that $M$ and $P$ have the same quality level $q_{M}=q_{P}=q$. Then, absent salience distortions, the decision maker values both objects at $q / 2$.

\footnotetext{
${ }^{3}$ The main results go through for complex goods having non-zero attribute values.
} 
As the decision maker is given the mug in the endowment stage, he evaluates $M$ against the status quo $(0,0)$ of not having it. The consideration set is $C^{e}=\{(q, 0),(0,0)\}$ and the average good is $(q / 2,0)$. By ordering, the quality of the mug is salient: $\sigma(q, q / 2)>\sigma(0,0)=$ 0 . The local thinker weighs by $1 /(1+\delta)$ the mug's quality for drinking and by $\delta /(1+\delta)$ its (zero) quality for writing, so that the weights add up to one. The mug's perceived value is:

$$
v^{L T}\left(M \mid C^{e}\right)=q \cdot \frac{1}{1+\delta}>q \cdot \frac{1}{2}
$$

The mug is overvalued because its quality is salient against the backdrop of not having it. Since in the endowment stage the local thinker's focus is on the mug's quality, this focus should also play a role when he subsequently considers whether to trade the mug. To capture this idea in a simple way, we assume that the mug's salience ranking in the endowment stage carries through to the trading stage with probability $\gamma$ : when $\gamma>0$ there is a warm glow of ownership in the trading stage.

In the trading stage, the decision maker must decide whether to trade his mug for a pen. The consideration set thus becomes $C^{t}=\{(q, 0),(0,0),(0, q)\}$, and the average good is $(q / 3, q / 3) .{ }^{4}$ As a result, the pen's quality for writing is not salient because:

$$
\sigma(0, q / 3)>\sigma(q, q / 3) \Leftrightarrow \sigma(0,1 / 3)>\sigma(1,1 / 3)
$$

which follows from homogeneity of degree zero. Due to diminishing sensitivity, the pen's complete lack of quality for drinking is more salient than its higher-than-average quality for writing, implying that at the trading stage the value of the pen is

$$
v^{L T}\left(P \mid C^{t}\right)=q \cdot \frac{\delta}{1+\delta}<q \cdot \frac{1}{2}
$$

Because the mug and the pen are perfectly symmetric goods, in the trading stage $C^{t}$ they both have a salient downside. However, accounting for the warm glow of ownership,

\footnotetext{
${ }^{4}$ Removing decision maker's status quo $(0,0)$ from $C^{t}$ does not substantially affect our analysis.
} 
the mug's average valuation in the second stage is:

$$
v^{L T}\left(M \mid C^{t}, C^{e}\right)=q \cdot \frac{\delta(1-\gamma)+\gamma}{1+\delta}
$$

The mug may also be undervalued relative to the rational case. However, as long as $\gamma>0$ it is valued more than the pen. As a consequence, the local thinker keeps it, exhibiting the endowment effect. ${ }^{5}$ If $\gamma=0$, the endowment effect disappears.

This mechanism can provide a context-based foundation for loss aversion based on the idea that the valuation of the goods we own is at least partly formed against the backdrop of not having them, while trades are valued by comparing exchange options. ${ }^{6}$ The first comparison induces us to focus on the best attributes of the goods we own, while the second comparison induces us to focus on either good's relative disadvantages. The combination of the two stages boosts the relative valuation of the goods we own. This perspective on the endowment effect makes several testable predictions:

i) If the good available for trade is an identical mug, $(q, 0)$, then $C^{t}=\{(q, 0),(0,0),(q, 0)\}$, and the average good is $(2 q / 3,0)$. Because $\sigma(q, 2 q / 3)>\sigma(0,0)$, the upside is salient both for the new mug and for the original one, so both are valued at (1). There is no endowment effect for identical objects. Similarly, if the new good is a better mug, $(2 q, 0)$, the decision maker likewise focuses on its upside and overvalues it. There is no endowment effect in upgrading. The endowment effect requires a trade-off between the endowed good and the new good.

ii) If the endowment is a bad $(-q, 0)$, then in the endowment stage the decision maker focuses on the bad's downside because $\sigma(-q,-q / 2)>\sigma(0,0)$. Given the option to trade the endowment with a different bad $(0,-q)$, he focuses on the upside 0 of the latter: by diminishing sensitivity and reflection $\sigma(0,-q / 3)>\sigma(-q,-q / 3)$. In the case of bads there is then a "cold glow" of ownership and people are overly willing to trade their lot. There is a reverse endowment effect for bads.

\footnotetext{
${ }^{5}$ Expression (3) can also be interpreted as the evaluation of a subject who averages between the salience rankings of the two stages.

${ }^{6}$ If the pen is sufficiently better than the mug, e.g. $q_{P}>q_{M} \cdot\left[1+\gamma \frac{1-\delta}{\delta}\right]$, the local thinker will trade the pen for the mug (even though the pen's downside is still salient). The coefficient in square brackets can be viewed as the loss aversion parameter.
} 
iii) If the endowment is a pen and a mug, the warm glow of ownership would apply to all goods. As a result, keeping the assumption of linear utility, the decision maker is no longer reluctant to exchange a mug for a pen (or vice versa) in the trading stage. Thus, there is no endowment effect for comprehensive endowments.

\section{Of Mugs and Bucks}

We now turn to the experimental evidence of a gap between decision makers' willingness to pay (WTP) and willingness to accept (WTA). Consider again the case of a mug of quality $q$. Here $q_{1}$ is the quality $q$ of the mug while $q_{2}=-p$ is its price disutility. The utility from the mug $(q,-p)$ is $q / 2-p / 2$.

In the endowment stage the consideration set is $C^{e}=\{(q, 0),(0,0)\}$ and the mug's upside is salient. In the trading stage the decision maker includes in the consideration set the option $(0, \mathrm{WTA})$ of obtaining his WTA, so $C^{\mathrm{WTA}}=\{(q, 0),(0,0),(0, \mathrm{WTA})\}$. As before, by diminishing sensitivity the downside of all options in $C^{\mathrm{WTA}}$ is salient. The decision maker's utility from $(0, \mathrm{WTA})$ is:

$$
v^{L T}\left((0, \mathrm{WTA}) \mid C^{\mathrm{WTA}}\right)=\mathrm{WTA} \cdot \frac{\delta}{1+\delta}<\frac{\mathrm{WTA}}{2} .
$$

The monetary gain is undervalued because the decision maker focuses on the loss of the mug. The value of the mug is equal to (3) as before. The decision maker's WTA equates (4) and (3) and is thus equal to:

$$
\mathrm{WTA}=q \cdot\left(1+\gamma \cdot \frac{1-\delta}{\delta}\right) .
$$

Consider now the decision maker's WTP for the mug. Because he is not endowed with the mug, he has no warm glow of ownership. He then determines his WTP in $C^{\mathrm{WTP}}=$ $\{(q,-\mathrm{WTP}),(0,0)\}$. Now the price and the quality of the mug are equally salient, so the decision maker states his correct valuation:

$$
\mathrm{WTP}=q
$$


Compare (5) to (6): in line with the endowment effect, there is a positive WTA-WTP gap, equal to $q \cdot \gamma \cdot(1-\delta) / \delta$. This gap is shaped by the warm glow of ownership $\gamma$, as well as by the extent of local thinking $\delta$.

Additional evidence for this mechanism is provided by by Kahneman et al (1991). Consider a decision maker who is not endowed with a mug, but is asked for his mug cashequivalent at the trading stage. He faces a problem identical to that of the endowed subject, namely, finding the price at which he is indifferent between receiving that price or the mug. Yet, due to the absence of warm glow $(\gamma=0)$, we predict that this subject's WTA is given by (6) and not by (5). This is consistent with Kahneman et al's (1991) findings.

Weaver and Frederick (2011) show that the WTA-WTP gap changes when subjects are provided with information about the mug's market price. The dependence of WTA and WTP on market prices is not in itself surprising. With a high market price $p_{M}>q$, a rational decision maker expecting to sell the mug in the market with probability $\alpha$ (and to keep it with probability $1-\alpha)$ values the mug at $\alpha p_{M}+(1-\alpha) q$. The value of the mug clearly increases in $p_{M}$, but of course there is no endowment effect. ${ }^{7}$ What needs to be explained is the persistence of the WTA-WTP gap, and its amplification with high market prices.

From the local thinker's perspective, information about market prices simply brings to his attention an alternative valuation of the mug besides consumption, namely the possibility of trading at the market price. Relative to the case of no reference prices, the local thinker's consideration set in the trading stage now includes the option $\left(0, p_{M}\right)$ of selling the mug in the market, $C^{t}=\left\{(q, 0),(0,0),(0, \mathrm{WTA}),\left(0, p_{M}\right)\right\}$. To determine WTA, note that also in this context the downside of each option is salient. Moreover, the mug's quality is boosted by $1 / \delta$ due to the warm glow of endowment, as in $(5)$ (where for simplicity we set $\gamma=1$ ). The decision maker's selling price is thus

$$
\mathrm{WTA}=\alpha p_{M}+(1-\alpha) q \cdot \frac{1}{\delta}
$$

When stating his WTP for a mug, however, the local thinker's consideration set is $C^{\mathrm{WTP}}=$ $\left\{(q,-\mathrm{WTP}),(0,0),\left(0, p_{M}-\mathrm{WTP}\right)\right\}$, which also takes into account the possibility of trading

\footnotetext{
${ }^{7}$ The probability $\alpha$ of trade is taken to be rational and exogenous, and may depend on the cost/ease of finding trading partners.
} 
the mug at market price, namely $\left(0, p_{M}-\mathrm{WTP}\right)$. Then, provided the market price is sufficiently high relative to $q,{ }^{8}$ the downside of each option is salient. In particular, the price WTP paid when buying the mug is very salient to the buyer. Thus, given an expectation $\alpha$ of re-selling the mug, the local thinker's buying price is

$$
\mathrm{WTP}=\left(\alpha p_{M}+(1-\alpha) q\right) \cdot \frac{1}{\alpha+\frac{1-\alpha}{\delta}}
$$

Equations (7) and (8) capture the WTA-WTP gap in the presence of reference prices. Two points can be noted. First, the gap arises whenever the local thinker is not certain about trading: for any $\alpha<1$ (and $\delta<1$ ) we have WTA $>$ WTP. ${ }^{9}$ When $\alpha=1$ the gap disappears as $\mathrm{WTA}=\mathrm{WTP}=p_{M}$, just as in the rational case. Second, consistent with Weaver and Frederick, the selling price is more sensitive than the buying price to $p_{M}$ when $p_{M}$ is high. In this regime the WTA-WTP gap increases with the good's market (or reference) price. A similar calculation shows that when $p_{M}$ is low relative to $q$, the selling price is less sensitive than the buying price to $p_{M}$. In this case, as $p_{M}$ becomes smaller the WTA-WTP gap increases, resulting in a $U$-shaped relation between WTA-WTP gap and $p_{M} \cdot{ }^{10}$

\section{Conclusion}

Unlike Prospect Theory, our model does not feature loss aversion, either in the utility or in the salience functions, which can both be symmetric in gains and losses [e.g. the salience function may satisfy $\sigma(-q, 0)=\sigma(q, 0)]$. We have shut down any mechanism involving loss aversion relative to expectations. The mechanism we propose is based on the novel ingredients of salience and context dependence.

Our approach highlights a fundamental difference between the context of absolute evaluation, in the endowment stage, and the context of comparative evaluation, in the trading

\footnotetext{
${ }^{8}$ Formally, this is true when $p_{M}>2 \cdot \mathrm{WTP}$, where WTP is given by (8). This follows from $p_{M}>q$ if trade is unlikely and $\delta$ is small.

${ }^{9}$ This is the case even if $\gamma=0$. The asymmetry between buying and selling arises at the trade stage: since downsides are salient, the buying price is relatively more salient for the buyer.

${ }^{10}$ This feature is also predicted by Weaver and Frederick, who provide suggestive evidence for it. To see how it arises in our model, note that when $p_{M}<q$, the owner of the mug never sells it in the market, and sets $\mathrm{WTA}=q /(1+\delta)$. The subject without a mug can try to buy it in the market. His consideration set is then $C^{t}=\left\{(q,-\mathrm{WTP}),(0,0),\left(q,-p_{M}\right)\right\}$ and so his buying price WTP decreases as $p_{M}$ goes to zero.
} 
stage. In the former, the decision maker focuses on the endowment's most extreme attribute (due to the ordering property of salience), whereas in the latter his attention is drawn to the alternative's downside (due to diminishing sensitivity), generating an endowment effect. In our view, what makes ownership special is the focus on the most attractive attributes of the goods one owns; there is no warm glow of ownership when these attributes are surpassed.

This intuition highlights a deep connection between the endowment effect and attitudes towards risk. In BGS (2011a) we showed how the same mechanism of salience can shed light on risk attitudes, whereby the decision maker is risk averse if he focuses on a risky lottery's downside, and risk seeking if he focuses on its upside. Similarly, here the endowment effect is due to an aversion to the alternative good generated by focusing on its downside. ${ }^{11}$ Moreover, just as BGS (2011a) show that salience generates a shift from risk seeking to risk aversion as lottery gains are reflected into losses, here we predict a reverse endowment effect for bads. Salience therefore provides a unified account of disparate puzzles such as the endowment effect, preference reversals, and the public health dilemma as the consequence of the same perceptual forces of diminishing sensitivity and ordering applied to different contexts of absolute and comparative evaluation.

\section{References}

[1] Bordalo, Pedro, Nicola Gennaioli and Andrei Shleifer. 2011a. "Salience Theory of Choice under Risk", NBER working paper 16387.

[2] 2011b. "Salience and Consumer Choice", Unpublished Manuscript, Harvard University.

[3] Brenner, Lyle, Yuval Rottenstreich, Sanjay Sood and Baler Bilgin. 2007. "On the Psychology of Loss Aversion: Possession, Valence, and Reversals of the Endowment Effect." The Journal of Consumer Research, 34(3): 369-376.

[4] Ericson, Keith and Andreas Fuster. 2011. "Expectations as Endowments", Quarterly Journal of Economics, 126(4): 1879-1907.

\footnotetext{
${ }^{11}$ This intuition can be formalized to account for recent evidence on an endowment effect for risk, as documented by Charles Sprenger (2010).
} 
[5] Heffetz, Ori and John List, 2011, "Is the Endowment Effect a Reference Effect?", NBER working paper 16715 .

[6] Kahneman, Daniel, Jack L. Knetsch, and Richard H. Thaler. 1991. "Experimental Tests of the Endowment Effect, and the Coase Theorem", Journal of Political Economy, 98(6): $1325-1348$.

[7] Kahneman, Daniel, and Amos Tversky. 1979. "Prospect Theory: an Analysis of Decision under Risk" Econometrica, 47(2): 263-292.

[8] Knetsch, Jack L. 1989. "The Endowment Effect and Evidence of Nonreversible Indifference Curves", American Economic Review, 79(5): 1277-1284.

[9] Koszegi, Botond and Matthew Rabin. 2006. "A Model of Reference-Dependent Preferences", Quarterly Journal of Economics, 121(4): 1133-1165.

[10] List, John. 2003. "Does Market Experience Eliminate Market Anomalies?", Quarterly Journal of Economics, 118(1): 41-71.

[11] Novemsky, Nathan and Daniel Kahneman. 2005. "The Boundaries of Loss Aversion", Journal of Marketing Research, 42(2): 119-128.

[12] Sprenger, Charles. 2010. "An Endowment Effect for Risk: Experimental Tests of Stochastic Reference Points", Stanford Working Paper.

[13] Thaler, Richard. 1980. "Toward a Positive Theory of Consumer Choice", Journal of Economic Behavior \&3 Organization, 1(1): 39-60.

[14] Tversky, Amos and Dale Griffin. 1991. "Endowment and contrast in judgments of wellbeing", in Subjective Well-Being, Fritz Strack, Michael Argyle and Norbert Schwarz eds. (Pergammon).

[15] Tversky, Amos and Daniel Kahneman. 1991. "Loss Aversion in Riskless Choice: a Reference Dependent Model", Quarterly Journal of Economics, 106(4): 1039-1061.

[16] Weaver, Ray and Shane Frederick. 2011. "A Reference Price Theory of the Endowment Effect", Unpublished Manuscript. 